\title{
Pression sociale et apparence physique
}

\author{
Jacques Janvier Rop's Okoué Edou \\ Faculté des Sciences de l'administration \\ Université du Québec à Rimouski
}

\section{INTRODUCTION}

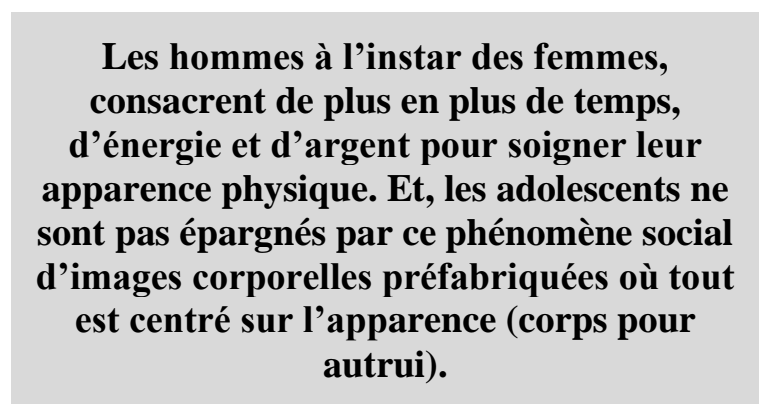

Depuis quelques années, un commerce de type nouveau à savoir celui de l'homme et de son apparence (physique) prend de plus en plus d'essor dans la société. Les masses médias (télé, journaux, publicité, internet, etc.) noient les populations d'images ou d'idées selon lesquelles l'homme peut être plus beau et avoir plus d'estime de soi au point que ceux qui choisissent de calquer leur vie sur cet impératif de perfection acceptent difficilement d'avoir un corps impuissant à satisfaire leurs exigences et encore moins de vieillir.

Dans ce cadre, les hommes à l'instar des femmes, consacrent de plus en plus de temps, d'énergie et d'argent pour soigner leur apparence physique. Et, les adolescents ne sont pas épargnés par ce phénomène social d'images corporelles préfabriquées où tout est centré sur l'apparence (corps pour autrui). Pour certains, la famille, les amis, les médias, bref la société tout entière expliquerait ce phénomène ou fait social au travers de la trop grande pression qu'elle exerce sur les individus.

Selon une psychologue clinicienne américaine : « sous les pressions sociales et familiales, les adolescents marinent comme les adultes dans l'autocuiseur de la performance, évalués et jugés à longueur de journée par leurs pairs, parents, entraîneurs, professeurs, etc. ${ }^{1} »$. Cette pression sociale sur les adolescents nord-américains les pousse jusqu'à recourir à la chirurgie plastique, à la liposuccion pour mieux ressembler aux modèles encensés par les médias.

Face à ce fait social, le sujet d'étude faisant l'objet de la recherche scientifique ici est «pression sociale et apparence physique des jeunes étudiants du programme sciences humaines du cégep Limoilou ».

Le cadre de référence choisi permet de mettre en exergue les théories sociologiques (fonctionnalisme, marxisme, interactionnisme) de la pression sociale et les concepts sous-jacents (corpsobjet, corps pour autrui, estime de soi...) à l'aune de l'influence sociétale sur un individu.

Sur la base des théories susmentionnées, il s'agit de savoir si la pression sociale a une influence (positive) sur l'apparence physique des jeunes étudiants du programme «sciences humaines » du cégep Limoilou. L'étude de la relation entre les phénomènes (pression sociale et apparence physique des jeunes étudiants) s'inscrit dans une approche théorique « pluraliste $»^{2}$.

Le devis de la présente recherche scientifique se veut explicatif ${ }^{3}$ au sens de simplement établir des relations d'association entre la "pression sociale » et l' " apparence physique » des jeunes étudiants du programme «sciences humaines » du cégep Limoilou. Il repose principalement sur trois techniques de collecte des données, à savoir l'analyse du contenu, l'analyse des statistiques et le sondage. 


\section{CORPS, APPARENCE ET ESTIME DE SOI}

Les premières théories du corps humain émanent des philosophes de la Grèce antique qui développèrent des doctrines des plus extrêmes sur le corps ou l'apparence physique. Certains (Socrate, Platon...) vont mépriser le corps ou n'en tiendront pas compte, préférant la noblesse de l'âme et de la pensée à la bassesse du corps et de la chair. D'autres (les épicuriens notamment comme Épicure, Lucrèce...) vont a contrario affirmer la prééminence du corps, notamment dans sa dimension sensuelle et situeront la grandeur de l'homme dans sa corporéité ${ }^{4}$.

\subsection{Le corps-objet ou le corps pour autrui sous la pression de la société}

Cette réflexion sur le corps humain et son apparence en est une bonne pour savoir si le corps est un corps-sujet (vécu de l'intérieur) ou plutôt, un corps-objet, visible de l'extérieur (morphologie, apparence), pouvant influencer notre comportement sous la pression de la société (parents, amis, médias, etc.).

Dans ce cadre, la «pression sociale » est l'influence exercée par un groupe sur chacun de ses membres aboutissant à lui imposer ses normes dominantes en matière d'attitude et de comportement.

L'« apparence $»^{5}$ qui vient étymologiquement du vocable apparentia est la manière dont une personne va se présenter, se percevoir, se voir, s'estimer. Le concept d' « estime de soi », proche de celle de personnalité, se définit comme une structure organisée des perceptions que l'individu se fait de lui-même, c'est-à-dire des perceptions de ses caractéristiques par rapport à son environnement. Il est multidimensionnel et peut intégrer le soi réel (façon dont le sujet se perçoit), le soi idéal (façon dont le sujet voudrait être), le soi social (façon dont le sujet pense que la société le perçoit) et le soi corporel ou physique (perception et évaluation du corps, particulièrement les capacités et l'apparence physiques).

La discrimination corps-sujet ou corps pour soi et corps-objet ou corps pour autrui permet de distinguer trois approches (théories) sociologiques du comportement de l'individu en so- ciété : le fonctionnalisme (théorie de l'intégration), le marxisme (théorie du conflit social) et l'interactionnisme.

1.2 Le fonctionnalisme et le marxisme comme théories macrosociologiques corroborant la thèse de l'influence de l'individu par la société

Le fonctionnalisme ${ }^{6}$ ou théorie macrosociologique de l'intégration s'est développé dans le siècle dernier en vue d'expliquer le fonctionnement global de la société. Il conçoit la société comme un ensemble de parties toutes interreliées dont certaines, nommées « institutions sociales », exercent une pression sur chacun des membres de la société et jouent un rôle précis dans l'équilibre social que celle-ci tente d'atteindre (la famille constitue l'une des premières institutions sociales). Aussi, pour que la société tende vers un certain ordre social, ses membres doivent partager les mêmes valeurs fondamentales.

Le marxisme élabore une théorie macrosociologique du conflit social entre deux classes à savoir, la bourgeoisie qui possède les moyens de production (usines, technologie, etc.) et les richesses d'une société, et le prolétariat qui n'a que sa force de travail à vendre pour assouvir ses besoins essentiels ou primaires. Selon K. Marx, la bourgeoisie régit, dans toute société, la production des idées et des valeurs nécessaires à son bon fonctionnement et à la libre circulation du commerce. Elle régule notamment les instruments idéologiques très puissants que sont les médias d'information et qui lui permettent d'assurer son pouvoir sur les classes laborieuses.

Les hommes s'accordent sur les caractéristiques corporelles contribuant le plus à la beauté masculine : l'apparence générale, la structure du corps, la répartition du corps, la répartition du poids, le visage, le teint et les dents. Un tel consensus est attribuable à la standardisation des canons esthétiques d'origine occidentale, à leur utilisation publicitaire (jeunesse, sport, santé, mode...) et à l'irruption télévisuelle des stars, mannequins et héros sportifs uniformisés dans les foyers. 
Globalement, il se dégage, pour les deux théories macrosociologiques susmentionnées, que l'individu subit de façon mécanique les contraintes du milieu dans lequel il évolue. Sur le plan qualitatif, les travaux relatifs à l'attrait esthétique du corps établissent l'influence déterminante de la beauté physique, faciale et corporelle sur l'évaluation d'autrui, les préférences, les attitudes et les comportements différentiels de l'entourage comme sur l'image de soi. «What is beautiful is good»? Les hommes s'accordent sur les caractéristiques corporelles contribuant le plus à la beauté masculine : l'apparence générale, la structure du corps, la répartition du corps, la répartition du poids, le visage, le teint et les dents ${ }^{8}$. Un tel consensus est attribuable à la standardisation des canons esthétiques d'origine occidentale, à leur utilisation publicitaire (jeunesse, sport, santé, mode...) et à l'irruption télévisuelle des stars, mannequins et héros sportifs uniformisés dans les foyers 9 . Sur le plan quantitatif, des travaux sur le conformisme et les influences sociales ont montré que les gens se conforment en moyenne à la majorité dans $37 \%$ des cas. Par ailleurs, $50 \%$ se conforment une fois sur deux. Une autre étude a montré que le conformisme augmentait avec la grandeur du groupe (1, 2, 3, 4, 8 ou 15 complices).

Tous ces travaux ont notamment expliqué le conformisme par les effets de l'influence des normes sociales qui amènent à se conformer par peur des conséquences négatives ou pour être davantage aimé ou accepté par le groupe. Les recherches quantitatives ont montré que les individus qui s'éloignent des normes d'un groupe sont souvent rejetés, haïs, ridiculisés...

\subsection{L'interactionnisme comme théorie microsociologique réfutant la thèse de l'influence de l'individu par la société}

A contrario de ces deux théories macrosociologiques, l'interactionnisme qui se veut microsociologique part du point de vue de l'individu et développe l'idée que chaque être humain, en association avec les autres (autrui), contribue à faire de la société ce qu'elle est. Il part du principe que chaque individu agit librement, et ce, dans un contexte social précis. Autrement dit, l'individu ne subit pas de manière mécanique les contraintes du milieu, mais construit plutôt son action en développant des raisonnements qu'il utilise dans la vie quotidienne.

Sur le plan qualitatif, les travaux menés par S. Moscovici ${ }^{10}$ se sont intéressés à la dynamique du conflit généré par la présence d'une source déviante minoritaire. Autrement dit, pour ce dernier, la minorité consistante peut, de par son style de comportement (manière dont les sujets se comportent et s'expriment dans l'interaction), influencer et changer une majorité. L'étude quantitative qui a corroboré ce changement de l'approche de l'influence dans l'histoire de la psychologie sociale est celle de l'expérience du bleu-vert des années 1960.

\section{CE QUE L'ON VOUDRAIT SAVOIR SUR LA RELATION ENTRE CORPS, APPARENCE ET ESTIME DE SOI}

Face à la dialectique entre les théories sociologiques, en s'appuyant sur les thèses macrosociologiques fonctionnaliste et marxiste, il est approprié de savoir si la société exerce une pression sur l'apparence (physique) des hommes.

L'étude de la relation entre ces deux phénomènes s'inscrit notamment dans une approche théorique «pluraliste » selon laquelle la pression sociale (PS) est susceptible d'influencer l'apparence physique (AP) des hommes. Autrement dit, selon cette approche, si la pression sociale exerce une influence sur l'apparence des individus, c'est parce que ces derniers se conforment ou se soumettent aux croyances, opinions, normes d'attitude et de comportement de la société. Toutefois, si de nombreux chercheurs se sont penchés, dans une approche pluraliste, sur le lien (positif) entre la pression sociale et l'apparence physique des hommes, cette problématique a rarement été abordée dans le cadre spécifique de Québec et surtout, sur les jeunes étudiants. Aussi, se pose-ton la question de savoir si les conclusions de ces chercheurs peuvent, en la matière, valoir dans le cas québécois et surtout, pour les jeunes étudiants $\mathrm{du}$ programme «sciences humaines » du cégep Limoilou? Autrement dit, n'est-il pas justifié, au regard d'un manque de travaux scientifiques en la 
matière sur la région de Québec, de mener de nouvelles recherches?

Dans ce cadre, la question spécifique est donc celle de savoir si la pression sociale a une influence (positive) sur l'apparence physique des jeunes étudiants du programme «sciences humaines » du cégep Limoilou.

C'est donc ici un problème de recherche qui est d'une pertinence ${ }^{11}$ ou d'intérêt majeur quant à sa contribution à la fois théorique, méthodologique et statistique. Son apport théorique concerne la contribution des théories psychosociologiques (fonctionnalisme, marxisme et interactionnisme) à

\section{LE CADRE DE RÉFÉRENCE}

Le cadre de référence de la recherche menée ici porte sur la relation entre la pression sociale définie comme l'influence exercée par un groupe sur chacun de ses membres aboutissant à lui imposer ses normes dominantes en matière d'attitude et de comportement (acquiescement, conformisme, etc.) et l'apparence physique (des jeunes étudiants) entendue comme la manière dont une personne va se présenter, se percevoir, s'estimer. Il repose sur la vérification d'association entre la pression sociale et l'apparence physique des jeunes étudiants du programme « sciences humaines » du cégep Limoilou via les concepts de corps-objet, d'estime de soi.

En effet, le sentiment de satisfaction corporelle pour autrui ou la société (corps-objet) lié aux concepts d'estime de soi va jouer un rôle important dans la relation entre la pression sociale et l'apparence des jeunes étudiants. Le niveau de satisfaction corporelle élevé va diriger ces derniers vers une apparence généralement acceptée par la société soit par acquiescement, conformisme, intériorisation.

Cette étude s'appuyant sur les résultats de travaux antérieurement menés sous d'autres cieux, l'existence de relation (positive) entre les deux variables est présumée ce, à l'aune des théories de l'intégration (fonctionnalisme), du conflit social (marxisme). la compréhension de l'influence du comportement de l'individu par la société.

L'apport méthodologique de cette étude consiste en l'opérationnalisation des concepts clés (pression sociale, apparence physique) en vue d'une meilleure observation sur le terrain des phénomènes en relation au travers des dimensions et indicateurs y afférents. Son apport statistique, lié à sa visée explicative, est de mettre en exergue une étude corrélationnelle (relation directionnelle) entre deux phénomènes (pression sociale et apparence physique des jeunes étudiants) à l'aide du test du khi-deux $\left(\chi^{2}\right)$ et du calcul du coefficient d'association (C).

La théorie macrosociologique de l'intégration (théorie fonctionnaliste de l'intégration) conçoit que l'individu subit et se soumet de façon mécanique aux contraintes du milieu dans lequel il évolue. Elle montre comment une société peut tendre vers un certain équilibre social en contraignant ses membres à partager les mêmes valeurs fondamentales sous peine de subir des conséquences négatives (rejet, ostracisme, haine, ridiculisation, etc.). Et, la théorie macrosociologique du conflit social (théorie marxiste du conflit social) conçoit également que l'individu subit et se soumet de façon mécanique aux contraintes du milieu dans lequel il évolue. Elle montre comment la bourgeoisie va contrôler les instruments idéologiques que sont les médias d'information pour influencer le comportement des classes laborieuses. Pour elle, ce sont les masses médias (publicité, irruption télévisuelle des stars, mannequins, héros sportifs et cinématographiques, etc.) régies par la bourgeoisie qui influencent justement l'apparence (structure du corps, répartition du corps, répartition du poids, etc.) des individus.

Aussi, relativement aux deux théories sociologiques susmentionnées, il va simplement s'agir, dans le cadre du programme « sciences humaines » du cégep Limoilou, de déterminer le degré de relation entre les deux phénomènes (pression sociale et apparence physique), et ce, sous l'hypothèse évidemment d'une influence (positive) de la pression sociale sur l'apparence (physique) des jeunes étudiants. 


\section{LA MÉTHODOLOGIE}

Le devis de la présente recherche scientifique se veut essentiellement explicatif au sens où son but est simplement d'établir des relations d'association entre la pression sociale et l'apparence physique des jeunes hommes du programme «sciences humaines » du cégep Limoilou. Il repose sur la détermination du milieu de l'étude, le contrôle des variables étrangères, la communication avec les participants, les instruments de mesure (opérationnalisation), le temps alloué à la collecte des données.

Relativement au milieu de l'étude (population, échantillon, taux de réponse, etc.), la population cible ${ }^{12}$ sur laquelle porte la recherche scientifique menée est celle des étudiants masculins inscrits au programme «sciences humaines » $d u$ cégep Limoilou. Cette population cible s'élève, à l'aune de la base de la population (liste exhaustive), à 496 étudiants ( $\mathrm{N}=496$ étudiants) en 2011. À partir de cette population cible, la collecte des données a porté sur un échantillon de 100 étudiants ( $\mathrm{n}=100$ étudiants) inscrits au programme des sciences humaines du campus de Québec.

L'observation a été naturelle au sens de s'être déroulée dans le milieu naturel du sujet observé (cégep de Limoilou) après obtention de la collaboration et de l'autorisation de la direction de l'entité.

Relativement au contrôle des variables étrangères, la méthode de recherche scientifique utilisée en vue d'atteindre l'objectif de recherche (confirmation de l'hypothèse de recherche) a été celle non expérimentale. Elle a reposé sur la technique de collecte de données dites d'enquête consistant en la réponse, par les sujets, aux formulaires de questions ou questionnaires à réponses fermées qui leur ont distribués ou envoyés. Plus exactement, cette technique d'investigation directe a conduit à la distribution par tri informatisé (procédé de sélection par lequel les éléments d'une population sont choisis au travers d'un programme informatique), le 19/10/2011 de $12 \mathrm{~h}$ à $14 \mathrm{~h}$, des formulaires de questions directement aux étudiants masculins et ce, à l'aune de la liste exhaustive des 496 étudiants $^{13}$ inscrits au programme «sciences humaines ». Selon ce rapport, le total d'étudiants inscrits au programme des sciences humaines (campus de Québec et de Charlesbourg confondus) était, en 2009-2010, de 1624 . Parmi ces derniers, $68 \%$ étaient inscrits au campus de Québec (soit 1104 étudiants) et $32 \%$ au campus de Charlesbourg (soit 521 étudiants). La répartition par sexe de ces étudiants étant, au campus de Québec, d'environ $45 \%$ pour les garçons et $55 \%$ pour les filles, il s'est donc dégagé un effectif total de 496 étudiants hommes (population mère, $\mathrm{N}=$ 496) d'où a été tiré un échantillon de 100 étudiants pour la collecte des données.

Toutefois, en raison de la possibilité d'influence de variables étrangères sur les résultats de l'étude, il a été procédé à l'adoption de certains moyens de contrôle de ces dernières. Le premier moyen de contrôle a été l'adoption d'un processus d'échantillonnage probabiliste aléatoire (hasard) simple et au resserrement des critères de sélection des éléments de l'échantillon en vue d'une baisse des fluctuations d'échantillonnage, de la marge d'erreur et donc, de l'obtention d'un échantillon homogène. Le deuxième moyen a consisté au choix d'une taille de l'échantillon $(\mathrm{n}=100)$ sur une population mère $(\mathrm{N}=496)$ du strict point de vue des caractéristiques (les éléments de cet échantillon présentent les mêmes caractéristiques que ceux de la population mère). Le troisième moyen a reposé sur un taux de réponse significatif (100\%: sur les 100 questionnaires distribués, la totalité est revenue remplie).

Relativement à la communication avec les participants, la technique d'investigation directe utilisée ayant conduit à la distribution directe des formulaires de questions par tri informatisé grâce à la générosité et la disponibilité de la Direction des Services de Technologie de l'Information (DSTI), il n'a pas été nécessaire de recourir à un personnel de collecte de l'information ni donc à sa formation. Le formulaire de questions envoyé aux participants était précédé d'un texte de présentation brève de l'auteur de la recherche, de la précision du sujet et des buts de la recherche.

Relativement à l'opérationnalisation (instruments de mesure ou analyse conceptuelle), les concepts utilisés dans cette recherche ne pouvant être mesurés directement, il a été procédé à leur 
opérationnalisation $^{14}$ en vue de les rendre aptes à mesurer les variables.

Dans ce cadre, les indicateurs utilisés dans cette recherche en vue d'une meilleure observation des

\section{RÉSULTATS ET DISCUSSION}

\section{$70 \%$ des personnes interrogées ont répondu ressentir de la pression sociale relativement à leur apparence physique.}

Le questionnaire envoyé aux sujets était composé de 10 questions relatives aux indicateurs (publicité, pression par les pairs, entrainement sportif intense, etc.) et phénomènes (pression sociale et apparence physique) arrêtés dans l'analyse conceptuelle. De cela, il est ressorti ces constats.

Premièrement, $70 \%$ des personnes interrogées ont répondu ressentir de la pression sociale relativement à leur apparence physique. (Tableau 1)

Deuxièmement, $68 \%$ des sujets interrogés estiment que l'apparence physique est, sous toutes ses formes (sport, habillement, etc.), importante contre $32 \%$. (Tableau 2)

Troisièmement, ceux qui ressentent de la pression sociale la perçoivent plus de leurs ami(e)s ou de la publicité et ce, à hauteur de $14 \%$. (Tableau 3)

Quatrièmement, $7 \%$ des sujets ont recours à de la chirurgie plastique. (Tableau 4)

Cinquièmement, $30 \%$ des informateurs accordent une importance aux suppléments. (Tableau 5)

Le croisement (combinaison) des questions 1 et 5 montre que $55 \%$ des jeunes hommes interrogés disent ressentir de la pression sociale relativement à leur apparence physique en sus de l'importance de cette dernière à leurs yeux. (Tableau 6)

L'analyse inférentielle du croisement des résultats des questions 1 et 5 a conduit au test d'indépendance/dépendance des variables étudiées via le calcul du khi-deux $\left(\chi^{2}\right)^{15}$ et du coefficient de contingence $(\mathrm{C})$. Ce test du khi-deux $\left(\chi^{2}\right)$ repose phénomènes étudiés ont été la publicité et la pression par les pairs pour le concept de pression sociale, et l'esthétie, l'entrainement sportif intense et l'habillement pour le concept d'apparence physique.

sur une procédure en sept étapes (hypothèses, marge d'erreur, effectifs théoriques, conditions du test, règle de décision, calcul du $\chi^{2}$, décision).

Dans ce cadre, il s'agit d'opposer l'hypothèse H0 : pas de lien entre pression sociale et apparence physique des jeunes hommes du programme des sciences humaines du cégep Limoilou (campus de Québec) à l'hypothèse $\mathrm{H} 1$ : lien entre la pression sociale et l'apparence physique des jeunes hommes du programme des sciences humaines du cégep Limoilou (campus de Québec). La marge d'erreur choisie était de $5 \%(\alpha=0.05)$. Le calcul des effectifs théoriques a donné ces résultats.

Les conditions de validité du test étaient réunies (tous les effectifs théoriques sont supérieurs à 5).

Avec le risque d'erreur $(\alpha=0.05)$, le degré de liberté $(d d l=(2-1) X(2-1)=1)$, le khi-deux tabulé était de 3,84 ( $\chi^{2}$ critiqueltabulé/théorique = $3,84)$. Dès lors, la règle de décision était de rejeter de H0 si $\chi^{2}$ calculé $>\chi^{2}$ tabulé $=3,84$. Le calcul du khi-deux de l'échantillon a donné ce résultat.

Dans ce cadre, la comparaison entre $\chi^{2}$ calculé et $\chi^{2}$ tabulé a donné à l'acceptation de $\mathrm{H} 1$ et au rejet de $\mathrm{H} 0$ $\left(\chi^{2}\right.$ calculé $=11,97>\chi^{2}$ tabulé $=3,84 \Rightarrow$ Acceptation de $\mathrm{H}_{1}$ et rejet de $\mathrm{H}_{0}$ ). Il y a donc une dépendance entre les variables pression sociale et apparence physique des jeunes étudiants. Autrement dit, la pression sociale influence bien (positivement) l'apparence physique des jeunes étudiants programme des sciences humaines du cégep Limoilou (campus de Québec).

Le test d'association (calcul du coefficient de contingence, $\mathrm{C})$ a donné ce résultat de 0,107 $(C=$ $\left.\chi^{2} / \chi^{2}+n=11,97 /(11,97+100)=10,7 \%\right)$. Avec une telle valeur de 0,107 près de 0 , le lien entre «pression sociale » et «apparence physique » des jeunes étudiants est plutôt faible. 
Tableau 1 - Perception de la pression sociale relativement à l'apparence physique

\begin{tabular}{|c|c|c|}
\hline Choix de réponses & Nombre de fois de la réponse choisie & Pourcentage \\
\hline $\mathbf{1}($ oui $)$ & $\mathbf{7 0}$ & $\mathbf{7 0} \%$ \\
\hline $\mathbf{2}$ (non) & $\mathbf{3 0}$ & $\mathbf{3 0} \%$ \\
\hline Total & $\mathbf{1 0 0}$ & $\mathbf{1 0 0 \%}$ \\
\hline
\end{tabular}

Tableau élaboré par l'auteur à l'aune des résultats du sondage effectué.

Tableau 2 - Importance de l'apparence physique sous toutes les formes

\begin{tabular}{|c|c|c|}
\hline Choix de réponses & Nombre de fois de la réponse choisie & Pourcentage \\
\hline $\mathbf{1}($ Oui $)$ & $\mathbf{6 8}$ & $\mathbf{6 8} \%$ \\
\hline 2 (Non) & $\mathbf{3 2}$ & $\mathbf{3 2} \%$ \\
\hline Total & $\mathbf{1 0 0}$ & $\mathbf{1 0 0 \%}$ \\
\hline
\end{tabular}

Tableau élaboré par l'auteur à l'aune des résultats du sondage effectué.

Tableau 3 - Pression sociale et origine de la pression

\begin{tabular}{|c|c|c|}
\hline Choix de réponses & Nombre de fois de la réponse choisie & Pourcentage \\
\hline 1 & $\mathbf{1 4}$ & $\mathbf{1 4} \%$ \\
\hline 2 & $\mathbf{5}$ & $\mathbf{5 \%}$ \\
\hline 3 & $\mathbf{6}$ & $\mathbf{6 \%}$ \\
\hline 3,4 & 2 & $\mathbf{2 \%}$ \\
\hline 4 & 14 & $\mathbf{1 4} \%$ \\
\hline $\mathbf{9 8}$ & $\mathbf{5 0}$ & $\mathbf{5 0} \%$ \\
\hline $1,2,3,4$ & 2 & $\mathbf{2 \%}$ \\
\hline 1,4 & 1 & $1 \%$ \\
\hline $1,2,3$ & 1 & $1 \%$ \\
\hline $2,3,4$ & 1 & $1 \%$ \\
\hline 2,4 & 1 & $1 \%$ \\
\hline $2,3,4$ & 1 & $1 \%$ \\
\hline 3,4 & 1 & $1 \%$ \\
\hline Total & 1 & $100 \%$ \\
\hline
\end{tabular}

Tableau élaboré par l'auteur à l'aune des résultats du sondage effectué.

Tableau 4 - Apparence et recours à la chirurgie plastique

\begin{tabular}{|c|c|c|}
\hline Choix de réponses & Nombre de fois de la réponse choisie & Pourcentage \\
\hline $\mathbf{1}($ Oui $)$ & 7 & $7 \%$ \\
\hline $2($ Non $)$ & 93 & $93 \%$ \\
\hline Total & 100 & $100 \%$ \\
\hline
\end{tabular}

Tableau élaboré par l'auteur à l'aune des résultats du sondage effectué.

Tableau 5 - Apparence et importance des suppléments

\begin{tabular}{|c|c|c|}
\hline Choix de réponses & Nombre de fois de la réponse choisie & Pourcentage \\
\hline $\mathbf{1}($ Oui $)$ & $\mathbf{3 0}$ & $\mathbf{3 0} \%$ \\
\hline $\mathbf{2}($ Non $)$ & $\mathbf{7 0}$ & $\mathbf{7 0} \%$ \\
\hline Total & $\mathbf{1 0 0}$ & $\mathbf{1 0 0 \%}$ \\
\hline
\end{tabular}

Tableau élaboré par l'auteur à l'aune des résultats du sondage effectué. 
Tableau 6 - Relation entre la pression sociale et l'apparence physique

\begin{tabular}{|c|c|c|c|}
\hline Pression sociale & \multicolumn{3}{|c|}{ Apparence physique (entraînement, habillement, esthétie) } \\
\hline & Important & Pas important & Total \\
\hline Oui & $\mathbf{5 5}$ & $\mathbf{1 5}$ & $\mathbf{7 0}$ \\
\hline Non & $\mathbf{1 3}$ & $\mathbf{1 7}$ & $\mathbf{3 0}$ \\
\hline Total & $\mathbf{6 8}$ & $\mathbf{3 2}$ & $\mathbf{1 0 0}$ \\
\hline
\end{tabular}

Tableau élaboré par l'auteur à l'aune des résultats du sondage effectué.

Tableau 7 - Effectifs observés et théoriques (pression sociale et apparence physique)

\begin{tabular}{|c|c|c|c|}
\hline Pression sociale & \multicolumn{3}{|c|}{ Apparence physique (entraînement, habillement, esthétie) } \\
\hline & Important & Pas important & Total \\
\hline Oui & $55 \mid 47,6$ & $15 \mid 22,4$ & $70(70 \%)$ \\
\hline Non & $13 \mid 20,4$ & $17 \mid 9,6$ & $30(30 \%)$ \\
\hline Total & $\mathbf{6 8}(68 \%)$ & $32(32 \%)$ & $100(100 \%)$ \\
\hline
\end{tabular}

Tableau élaboré par l'auteur à l'aune des résultats du sondage effectué.

Tableau 8 - $\chi^{2}$ calculé à partir des effectifs observés et théoriques

\begin{tabular}{|c|c|c|}
\hline O & T & $(\mathbf{O}-\mathbf{T})^{2} / \mathbf{T}$ \\
\hline 55 & 47,6 & 1,15 \\
\hline 13 & 20,4 & 2,68 \\
\hline 15 & 22,4 & 2,44 \\
\hline 17 & 9,6 & 5,70 \\
\hline
\end{tabular}

Tableau élaboré par l'auteur à l'aune des résultats du sondage effectué.

\subsection{Discussion : interprétation des résultats et les limites de l'étude}

Les résultats tirés du test du khi-deux constituent une réponse valable. Cette situation confère une certaine validité interne au travail mené ici au sens de la compatibilité entre ces résultats statistiques et l'hypothèse de recherche adoptée à l'aune des théories macrosociologiques du fonctionnalisme et du conflit social marxiste.

Dans ce cadre, ces résultats viennent confirmer ceux des études antérieures menées sous d'autres cieux confirmant le lien positif entre la pression sociale et l'apparence physique à l'aune des théories macrosociologiques de l'intégration et du conflit social.

Selon l'étude corrélationnelle menée ici, les résultats recueillis $\left(\chi^{2}\right.$ calculé $=11,97>\chi^{2}$ tabulé $=$ 3,84 ) confirment la présence d'une signification statistique malgré le faible lien d'association (C). Ainsi, ces résultats impliquent le rejet de l'hypothèse $\mathrm{H} 0$ et l'acceptation de l'hypothèse $\mathrm{H} 1$ de corrélation entre les variables non due au hasard. Ils confirment la validité interne du cadre de référence (confortation des prévisions émises sur ce dernier) au sens de la compatibilité entre le cadre de référence théorique et l'analyse statistique menée à l'aide du test du khi-deux.

Toutefois, le faible degré d'association entre les variables laisse présumer de l'absence d'autres variables pertinentes que la pression sociale qui influenceraient également l'apparence physique des jeunes étudiants du programme «sciences humaines » du cégep Limoilou (campus Québec).

Plus précisément, le faible degré d'association entre les variables, la faible taille de l'échantillon et le faible taux de sondage $(n / N=100 / 496=$ $20,16 \%)$, l'absence de variables pertinentes autres que la pression sociale pourraient justifier la faible validité externe des résultats obtenus (limite du travail). Dans ce cadre, il serait judicieux de répéter l'étude dans d'autres contextes. Par exemple, une étude économétrique de l'apparence physique (AP) des étudiants sur la pression sociale (PS) pourrait 
être conduite sur une chronique donnée via ce modèle : $\mathrm{AP}_{\mathrm{t}}=\mathrm{aPS}_{\mathrm{t}}+\mathrm{AP}_{0}+\mathrm{U}_{\mathrm{t}}$ avec $\mathrm{AP}=$ apparence physique $=$ variable expliquée $=$ variable endogène; $\mathrm{a}=$ coefficient de régression de AP sur PS; $\mathrm{PS}=$ pression sociale $=$ variable explicative $=$ va riable exogène; $\mathrm{AP}_{0}=$ apparence physique incompressible $=$ constante non nulle et $\mathrm{U}=$ résidu .

Somme toute, le choix du processus d'échantillonnage aléatoire simple, le taux de réponse $(100 \%)$ et le test du khi-deux $\left(\chi_{\text {calculé }}^{2}=11,97>\chi^{2}\right.$ tabulé $=3,84$ ) confèrent une certaine validité externe aux résultats obtenus au sens de permettre une certaine généralisation de ces derniers à toute la population cible des étudiants «programme des

\section{CONCLUSION}

Cette étude démontre qu'il y a une influence positive de la «pression sociale» sur l'«apparence physique » des jeunes étudiants du programme «sciences humaines » du cégep Limoilou, (campus de Québec). La mise en exergue de ce lien positif entre les deux variables étudiées s'est appuyée sur les travaux antérieurement menés et sur les théories fonctionnalistes de l'intégration et marxiste du conflit social selon lesquelles l'individu subirait de manière mécanique les contraintes de la société dans laquelle il évolue.

Conformément à l'hypothèse de recherche émise à l'aune notamment des théories fonctionnalistes de l'intégration et marxiste du conflit social, les tests statistiques d'indépendance $\left(\chi^{2}\right)$ et d'association $\left(\mathrm{C}=\chi^{2} / \chi^{2}+\mathrm{n}\right)$ ont, au regard de leurs résultats, globalement conclu à l'existence d'un lien (positive) ou dépendance, même si faible, entre les variables étudiées $\left(\chi^{2}\right.$ calculé $=$ $11,97>\chi^{2}$ tabulé $\left.=3,84\right)$. Autrement dit, la «pression sociale» exerce, du point de vue statistique, une certaine influence (positive) sur l'«apparence physique» des jeunes étudiants du programme «sciences humaines » du cégep de Limoilou (Campus de Québec). De la sorte, ces résultats statistiques confèrent une certaine prééminence des théories de l'intégration et du conflit social sur celle interactionniste.

Mais, la valeur du coefficient de contingence $(10,97 \%)$ symbolise un lien (positif) plutôt faible entre la «pression sociale » et l'«appa- sciences humaines » du cégep Limoilou (campus de Québec). Mais, cette généralisation des résultats connaît un léger bémol, celui de ne pas reposer sur un échantillon très représentatif de la population mère au niveau de la taille (cette dernière aurait de manière optimale dû être de 217 étudiants pour une population cible de 496 étudiants masculins que compte le programme des sciences humaines du campus de Québec). Malgré ces limites, c'est une étude scientifique qui contribue tout de même à l'avancement de la recherche fondamentale, et ce, notamment dans le domaine de la sociologie, de la psychologie ou de la psychosociologie au niveau de la ville (région) de Québec.

rence physique »des étudiants et sous-tend des limites à ce travail.

Ces limites au travail de recherche scientifique menée seraient relatives à la faible taille de l'échantillon $(\mathrm{n}=100$ étudiants) relativement à la population mère $(\mathrm{N}=496$ étudiants), au faible taux de sondage $(\mathrm{n} / \mathrm{N})$, à l'absence de variables explicatives pertinentes autres que la pression sociale, etc. Dans ce cadre, elles expliqueraient la faible validité externe (généralisation) ${ }^{16}$ des résultats de ces travaux. Malgré ces limites, c'est une étude scientifique qui contribue tout de même à l'avancement de la recherche fondamentale dans le domaine de la sociologie, de la psychologie ou de la psychosociologie au niveau de la ville (région) du Québec.

\section{BIBLIOGRAPHIE}

${ }^{1}$ Defoy, N. (2001). Perfection fatale, Guide ressources, p. 1.

${ }^{2}$ Mace, G. et Pétry, F. (2000). Guide d'élaboration d'un projet de recherche, PUL, pp. 25-33.

${ }^{3}$ Fortin, M.-F., Côté, J. et Fillion, F. (2006). Fondements et étapes du processus de recherche, Canada, Chenelière Éducation, chapitre 10, pp. 174-177.

${ }^{4}$ Damak, L. (1997). Corps du consommateur et design du produit: recherche d'une similarité ou d'une complémentarité?, Cahier ${ }^{\circ} 257$, Centre de recherche DMSP, p. 4.

${ }^{5}$ Robert, P. (2010). Le nouveau Petit Robert de la langue française, Le Robert, Paris, p. 116.

${ }^{6}$ Campeau, R., Sirois, M. et Rhéault, M. (2009). Initiation à la sociologie: Individu et société, $4^{\mathrm{e}}$ édition, Chenelière Éducation, pp. 54-59. 
${ }^{7}$ Dion, K. K., Berscheid, E. et Walter, E. (1972). What is beautiful is good, Journal of Personality and Social Psychology, 24, pp. 285-290.

${ }^{8}$ Brodsky, C. M. (1954). A study of norms of bodyform - behavioral relationships, Washington: catholic University of America Press.

${ }^{9}$ Bruchon-Schweitzer, M. et Maisonneuve, J. (1976). Aspects esthétiques et iconiques du corps, in $\mathrm{R}$. Francès (Ed.), Psychologie de l'art et de l'esthétique, PUF, Paris, pp. 235-275.

${ }^{10}$ Moscovici, S. (1979). Psychologie des minorités actives, PUF, Paris, pp. 121-152.

${ }^{11}$ Fortin, M.-F. (1996). Le processus de recherche: de la conception à la réalisation, Decarie, pp. 89-98.

${ }^{12}$ Huot, R. (2003). Méthodes quantitatives pour les sciences humaines, $2^{\mathrm{e}}$ édition, PUL, pp. 10-29.

${ }^{13}$ Cégep de Limoilou, Rapport annuel 2009-2010.

${ }^{14}$ Angers, M. (2009). Initiation pratique à la méthodologie des sciences humaines, CEC, pp. 22-37.

${ }^{15}$ Simard, C. (2008). Méthodes quantitatives Approche progressive pour les sciences humaines, $4^{\mathrm{e}}$ édition, Modulo, pp. 232-243.

${ }^{16}$ Baillargeon, G. (2009). Statistique appliquée pour les sciences de la gestion et les sciences économiques, $3^{\mathrm{e}}$ édition, Les Éditions SMG, p. 313. 